\title{
The mental health between epigenetics and individual beliefs
}

\author{
Dramnescu Marin \\ Bucharest Academy of Economic Studies, Bucharest, Romania
}

\begin{abstract}
Mental health is an integrative concept that is not limited to dysfunctions or accentuations of psychic processes or mechanisms of thought. The research effort focused on the idea that mental health is a functional optimum found at the intersection of cellular behavior, the physical environment, the external environment, with all its subtypes, the environment in which the individual manifests itself and the subjective, psychological environment, dominated mainly by unconscious behavioral routines, beliefs, values, and ultimately individual perspective on life. Mental health represents and manifests itself as an emerging process resulting from the correlated functioning of the biological, physiological, and in particular cellular mechanisms, the various, random and / or permanent influences and stimuli of the physical, social and professional environment and the superior motivational structures of the type of beliefs and individual perspective on life. Epigenetics is a contemporary discipline derived from genetics that includes the environmental context as an important part of heredity. Currently, this discipline strongly influences a variety of areas, including medicine, psychiatry and psychology.
\end{abstract}

Keywords. Mental Health, Cell Behavior, Epigenetic Approach, Beliefs.

\section{Introduction}

It is unanimously accepted that the becoming of a person is an effect of the interaction among genetic factors, environmental factors and educational factors. Their share in the individual's development differs according to the stage of the development, but their influence on mental health in particular and on the formation of personality in general is permanent and becomes manifest throughout the ontogenetic.

The attempt to understand how these factors influence the construction of personality, as a flexible and permanently adaptable structure to the environment, whether externally, internally or subjectively, has enabled more or less rigid positions, emphasizing either genetic or hereditary use, or the role of the environment in the ontogenetic becoming of the individual, or the importance of education, as an essential vector in the multidimensional formation of the individual.

In line with these beliefs, for example, more than half a century, and between 1880 and 1940, it was believed and constantly attempted to demonstrate that heredity determines race, mental health, behavior and intelligence, or that it is responsible for most dysfunctions either biological or psychological.

But the concepts of heredity and the environment as contrasting terms were introduced for the first time by Francis Galton in his English Men of Science: Their Nature and Nurture (Galton, 1874). Galton's approach was favorable to heredity, arguing that "no enabling environment can overcome the predispositions of an unhealthy physique, an intellectual deficit or a predisposition to brutality." (Galton, 1874, p.18) Following these ideas, Nature reports that "a higher number of studies suggest that biology exerts a significant influence on beliefs and behavioral patterns open to political attitudes, suggesting that "genes may have an impetus on people's attitudes when discussing abortion, immigration, the death penalty, and pacifism. (Buchen, 2012)

Based on these approaches, subsequent debates and research on human behavior in general have been dominated and driven by various conceptual pairs such as hereditary and behavioral, innate and acquired, or genetics and the environment. (Buchen, 2012) However, in the public discourse, the heredity-environment dichotomy remains unchanged, despite the efforts made by biologists through declarations over the past nine decades that this combination of words is redundant and confusing. Scientific papers, studies or scientific articles that have a heredity and the environment in the title, or which relate to the research on the interrelationships between the two concepts, are more and more common. 


\section{Epigenetics and mass media}

Epigenetics is a contemporary discipline derived from genetics that includes the environmental context as an important part of heredity. Currently, this discipline strongly influences a variety of areas, including medicine, psychiatry and psychology (Hollar, 2016). Taking into account the impact it has in these areas, epigenetics deeply influences the education sciences by decrypting the links between genetics and neuroscience (Mulder, Rijlaarsdam, \& Van IJzendoorn, 2017)

Epigenetics is associated with the long-term evolution of human development and physical and mental health. It is therefore essential to better understand the links between epigenetic processes and genderenvironment interaction, early development and education, and its impact on mental health.

Epigenetics is a branch of genetics that studies the variation of phenotypic traits that are caused by aspects of the environment, these changing the behavior of genes and affecting how cells decode them, that is, epigenetics is the science that studies gene change by the things that happen in life everyday - so we can understand a series of phenomena, things or situations that can be applied individually in order to ensure a quality life

The purpose of epigenetics is to investigate how much of the behavioral variation of a population sample depends on genetic variation, using studies on twins and adopted individuals (Plomin et al. 2013, p. 21).

The popularity of genetic language in day-to-day communication is rooted in the publication in 2005 of the results of the Human Genome Project, a project launched in 1990 to sequentially identify the sequence of over 3 billion pairs of nitrate bases constituting the human genome, approximately 25,000 genes in the human genome both physically and functionally. The objectives proposed in the Human Genome project were extremely ambitious. In the traditional biological thinking system, it is believed that the body needs a gene to provide the mock-up of each of the over 100,000 proteins available to the body. To this is added at least 20,000 regulatory genes which determine the activity of the genes encoding the proteins. Consequently, it was concluded that the human genome should contain at least 120,000 genes, located in the twenty-three pairs of chromosomes. It has been discovered, however, that the entire human genome is formed of only about 25,000 genes (Pennisi, 2004). For example, the Caenorhabditis elegans worm has a body built on a precise pattern consisting of exactly 969 cells and a simple brain with about 302 cells. The genome of Caenorhabditis has about 24,000 genes, while the human body, consisting of over fifty billion cells, contains only with 1500 genes more than the non-invertebrate microscopic worm which barely has a thousand cells. Drosofila melanogaster has 15,000 genes (Blaxter, 2003). Thus, the mite, which is much more complex than the Caenorhabditis worm has 9,000 genes less. As far as rodents are concerned, the results of comparative research projects show that humans and rodents have approximately the same number of genes! (Bruce, 2017, p. 66-68) There is the temptation to refer to the human genome as the "instruction manual", forming the representation of a human body that could be assembled using the components and instructions provided (Alexander D., 2013, p. 16).

This situation is exaggerated by the media that unveils the discovery of new genes involved singularly and declared responsible for various behaviors: "a study made on adolescents reveals the gene for happiness" (New Scientist), "The Science of Stress - Does Your Child have the " Worry " Gene? "(The Times) and so on.

In fact, the gene is just a segment of DNA encoding a protein or RNA regulatory molecule. There is no gene coding any kind of behavior in any living organism, not to mention the human body (Alexander, 2013, p.19). The problem of such a dichotomous language is that it promotes and reflects a fragmented image of the human being in which genetic factors and environmental factors are in opposition, excluding interdependencies between them. The accessibility of information and the vulgarisation of these through the media lead to endless "debates about hereditary factors vs. the factors acquired"as if the two categories of factors were in competition to assert either the supremacy of heredity or the environment. Contemporary biology does not support the idea that genes are "a manual of procedures" that determine the becoming and ultimately lead to the destiny of the individuals.

\section{Genetics and the environment}

Minimizing the importance of the environment in relation to heredity has caused an exacerbation of genetic determinism under the belief that genes "control" the biological system. This conviction not only led to a misallocation of research funds but also changed the way life is understood and appreciated in general. In the 
situation where there is a belief that genes control life and that genetic inheritance from the moment of conception is definitive, individuals can be considered victims of heredity by directly influencing their mental health (Bruce, 2017, p. 67)

Since the beginning of the genetics era, programming has been created through formal education to accept that individuals are subject to the power of their genes. Thus, a permanent fear was induced in the form of a continuous stressor, that one day the genes will activate at some point in the form of incurable diseases such as cancer or schizophrenia as in the case of mother, brother, sister's father, etc.

According to this type of understanding, many people justify their poor health not by a combination of mental, physical, emotional and spiritual causes, but by the non-synchronization of the bio-chemical mechanics of their body. On this background, for example, if one finds that some children are less receptive to adult control then medication is used to correct some supposed chemical imbalances, avoiding investigating what actually happens in the body, the psyche, the beliefs and their spirit.

Certainly, some Huntington-type diseases, beta-thalassemia and cystic fibrosis can be fully attributed to a single faulty gene. But single gene disorders occur in less than $2 \%$ of the population. But diseases such as diabetes, heart disease and cancer are not the result of a single gene but of complex interactions between several genes and environmental factors (Bruce, 2017, p. 67). The media information claiming that there are genes that are responsible for many ailments ranging from depression to schizophrenia have to be taken with reluctance.

Many researchers have identified various links between different genes and different diseases but they have rarely found that a trait or disease is caused by a single gene. Confusion occurs when the media repeatedly distorts the meaning of two verbs: correlating and causing. One is something to relate to a disease, to be correlated with a dysfunction, and another is to cause, to determine a disease. Causing a disease involves an action that supposes targeting and control (Nijhout,1990).

What does the genes activate? The answer was expressed in 1990 in the work Metaphore and the Role of Genes and Development (Nijhout,1990). The author argues that the idea of genes that control biological systems has been repeated so often and so long that the researchers have forgotten that it is just a hypothesis and not a truth. In reality, the idea that genes control biological systems is a supposition that has never been proven and is undermined by the latest scientific research (Nijhout,1990).

Following research in organic chemistry, it has been discovered that cells are composed of four types of molecules: polysaccharides (complex sugars), lipid (fat) nucleic acids (DNA and RNA) and proteins. Although normal cell function requires all four molecular types, for the living organisms, proteins are the fundamental component. Mainly cells are an assembly of units that produce proteins. Thus a way to understand a trillion cell body is given by a representation of a protein product tool. More than 100,000 types of protein are needed for the body to function.

In the last decade, research in epigenetics has established that gene transfer patterns through genes are not definitive at birth. Genes do not provide predestination. Environmental influences, including diet, stress and emotions, can alter these genes without modifying their basic layout. Moreover, recent studies in epigenetics have revealed that these changes can be passed on to future generations (Reik and Walter, 2001, p. 21-23) just as DNA patterns are transferred through the double spiral (Surani, 2001, p. 122). The discoveries in the field of epigenetics have extended and nuanced discoveries in genetics. After 1940, biologists have dealt with DNA isolation inside cells to study genetic mechanisms.

During this enucleation process, the nucleus was extracted from the cells and the chromosomal content formed by $50 \%$ of the DNA and $50 \%$ of the regulatory proteins removed. But research has not focused on proteins and their role, they focus only on DNA. The results of the research were so incomplete and flawed because, in the chromosome, DNA forms the nucleus, but the proteins cover it, making it impossible to read. When genes are covered by protein, DNA information cannot be read. It becomes available for reading by separating the DNA double stranded protein by a signal from the environment. Or as a result of activating a belief. Once the DNA is unveiled the cell makes a copy of the exposed gene. As a result, gene activity is controlled by the presence or absence of regulatory proteins which in turn are controlled by environmental signals. In other words, the flow of information is triggered by an environmental signal, it goes on to a regulatory protein, reaches DNA, RNA, and finally a protein. From this angle, the contribution of the environment is impossible to be measured (Dennis, 2003, p. 687). 
What is inherited from parents is not simple DNA, which can do nothing alone, but a complex system of DNA and RNA, proteins and nutrients that work together to regulate the growth and division of a cell. The human ovule before fertilization contains at least 3,000 different proteins and thousands of RNA molecules involved in gene expression (e.g., how genes are activated or inactivated). All of these components are integrated to organize the development of the fetus. The genes work and are influenced by signals from the extracellular environment. In the zig-zag state, DNA does not trigger development, but inherited proteins from the mother are the ones that determine what kind of DNA genes are to be activated or inactivated. Proteins are the elements that cause genes to produce the integrated complexity of life (Alexander, 2013, p. 20).

\section{Heritability and hereditary}

The heritability of a particular feature is a concept that is often misunderstood or misinterpreted. Until 1940 , heritability was synonymous with inheritance. Since then, it has gained a new, technical, more difficult to understand meaning. Understanding this concept explains why the media misreport genetic discoveries. Heritability is defined as the proportion of the variance of a trait that can be attributed to genetic variation in a population sample. Heritability can be expressed as a percentage, and this is mistakenly interpreted as referring to the share of the inheritance of a feature, when in fact this percentage represents the total percentage of variance in a population sample.

Heritability or heredity refers to sets of genetic variants we receive from our parents and refers to individuals. Heritability in its technical sense refers to the level of relevant genetic variation within a population. Unfortunately, this word often passes from one sense to another even within the same text, so when it comes to the value of $50 \%$ of the credibility it gives the impression that a particular feature of an individual is caused in proportion of $50 \%$ of its genes and 50\% of its growing environment, which is not the true meaning of hereditability.

\section{Epigenetics and mental health}

The perspective that epigenetics offers on understanding how mental health is impaired or strengthened becomes coherent and integrative. The mental health of the individual is dependent on interdependencies and inter-influences between genetic, environmental and educational factors.

Mental health is an integrative concept that is not limited to dysfunctions or accentuations of psychic processes or mechanisms of thought. Mental health is a functional optimum at the intersection of cellular behavior, the intra and extracellular environment, the physical environment, the external environment with all its subtypes, the environment in which the individual manifests, the type of diet and the subjective, psychological environment, mainly dominated by unconscious behavioral routines, emotions, feelings, beliefs, assumed values, and ultimately individual perspective on life.

Mental health represents and manifests itself as an emerging process resulting from the correlated functioning of the biological, physiological, and in particular cellular mechanisms, the various, random and / or permanent influences and stimuli of the physical, social and professional environment and the superior motivational structures of the type of beliefs and individual perspective on life.

In recent years, a lot of new information has emerged about epigenetics, which allows a clearer understanding of how development trajectories can have adaptive or unadjusted endpoints. Experiences and environmental exposures act on genes such as a dimmer switch to increase or decrease gene expression, the amount of gene product (RNA, protein) that generates in response to a particular environment. It is believed that this regulates neurodevelopment underlying learning, behavior, as well as mental and physical health.

In the process of development, the brain is vulnerable to the negative effects of social exposures of the environment, especially during its early development. In contrast, careful, stimulating and sensitive adult care plays an important role in the harmonious development of children. It should be noted, however, that not all children react in the same way to early experiences, whether positive or negative. Individual differences with hereditary emphasis may be the explanation behind different responses to environmental stimuli. The effects of the experience on modifying genetic expression can also be passed on to the next generation. Individual experiences and beliefs affect the extracellular environment through hormonal involvement and which stimulate in an implicitly specific way cell and genome functioning as well as the whole development process early on. 
Care, stability and love help build a solid foundation for brain development, ensure an optimal level of physical and mental health throughout life, normal learning and social and emotional development. Similarly, adverse environments and distorted adult relationships, including poverty, abuse, neglect, stress and trauma, can also change the expression of genes involved in the development and regulation of the nervous system. The risks and effects of negative experiences from childhood are the highest among those living near, below, or below the poverty line.

While many molecular mechanisms control gene expression, epigenetic processes provide a pertinent and innovative argumentation on how and in what social conditions the genes and the environment intersect. Evidence from a variety of studies suggests that epigenetic changes lead to many synaptic changes. For example, stress-related psychological states, suicidal ideation and attempts, depression, post-traumatic stress disorder, schizophrenia, and various brain changes due to psychoactive and antipsychotic drugs have led to epigenetic changes. Epigenetic processes can also transmit risks and disturbances from one generation to another, still underdeveloped and validated. Risks and protective factors can be passed from parents to children through behavioral and social factors or eventually through inherited epigenetic traits.

Individuals develop throughout their life various ideas and principles of life that ultimately influence their behavior, how to act and think. These ideas, heavily rooted in the human psyche, gain strength and become beliefs (beliefs). Individual beliefs and weltanschauung of each individual are formed throughout ontogenetic development as a result of the environmental influences they have to deal with or as a result of their own experiences and the interpretation of these experiences. Another way of forming and imposing them is given by knowledge. Knowledge is the main way to control the constraints of the environment and ensure professional and social progress, as well as the stability of mental health. Individual beliefs can also be the product of the results obtained. Individual success can fuel confidence and the conviction that it can be repeated. Last but not least, individual beliefs can become stable with imagination. This is possible because, in its functioning, the brain does not distinguish between imagination and reality, so that everything that is transmitted to it through thoughts or images tends to be materialized or translated into experience.

Convictions or beliefs alter the extracellular environment by releasing hormones (cortisol adrenaline, histamine, testosterone, estrogen, dopamine, vasopressin, oxytocin, serotonin). Cellular behavior is modeled by their reception at cell membrane level, which promotes physical or mental illness or physical recovery.

\section{Conclusions}

From the very beginning of development, genes exist simultaneously in two environments: the micromedium in the cell and the macromedia outside the cell, primarily the mother, then the growing fetus. DNA and these two types of environment (intracellular and extracellular) interact from fertilization. Many complementary processes take place in parallel, undermining the language of causal relationships.

The child's brain is not a miniature version of the adult brain but a system that self-organizes and assembles correctly if environmental inputs (light, sound, language, touch) are available at the right time. Continuing along the trajectory in adulthood development, the integrated interaction between genes, micromedium and macromedium becomes more controversial.

The growing individual makes essential choices about lifestyle, choices that bring epigenetic changes to DNA, activating and inactivating genes. If the genome is the hardware of the inheritance, then the epigenetic regulation is the software, the changes being kept in the cell division. Some can be inherited even over two generations. Epigenetic regulation provides an essential communication path that starts from macromedium through the micromedium and reaches the genome. The lifestyle choices we take can be passed on to the next generations.

The integrated approach, that of the development is that $100 \%$ of the phenotype of any complex organism involves genetics and $100 \%$ involves the environment. When we are born neither are we like "blank sheets of paper" nor are we the product of a manual of genetic instruction.

\section{REFERENCES}

1. McMahon B. (2013), Body and Soul, in The Times, 9 March, p.4

2. Blaxter M. (2003), Two worms are better than one, in Nature 426, p. 395-396. 
3. Bruce H. L., Biologia credinței, Ed. For You, București 2017

4. Denis A., (2013), Genes, Determinism and God, Cambridge Press, Volume 22, No 4, 2013,

5. Dennis C. (2003), Altered States in Nature, 421, p. 686-688,

6. Chakravarti A., and Little P,. (2003), Nature, nurture and human disease, in Nature, 421, p.412-414,

7. Cloud J., (2010), Why Your DNA Isn't Your Destiny, in Time.

8. Galton F., (1874), English Men of Science: Their Nature and Nurture, Macmill \& Co, London.

9. Goodman, L. (2003), Making a Genesweep: It's Official! in Bio-IT World.

10. Nijhout H.F., (1990), Metaphore and the Role of Genes and Development, in Bioessays, 129(9), p.441-446

11. Hollar, D. (2016). Epigenetics, The Environment, and Childrens Health Across Lifespans, Berlin: Springer.

12. Buchen L., (2012) Nature, 490, pp.466-468

13. Mulder, R. H., Rijlaarsdam, J., and Van IJzendoorn, M. H. (2017), DNA Methylation: a mediator between parenting stress and adverse child development? in Parental Stress and Early Child Development Adaptive and Maladaptive Outcomes, eds K. Deater-Deckard and R. Panneton (Berlin: Springer International Publishing), p. 157-180.

14. Pennisi, E., (2004), Researchers Trade insights About Gene Swapping, Science 305, p. 334-335. Pearson, H., Geneticists play the numbers game in vain, in Nature 423, p. 576,

15. Plomin R., et al, (2013) Behavioural Genetics, Worth Publishers, 6th edn, p 21

16. Reik. W and Walter J., (2001), Genomic imprinting: Parental Influence on the Genome, in Nature Reviews Genetics, 2, p. 21-23

17. Surani M.A., Reprogramming of genome function trough epigenetic inheritance, in Nature, 414, p. 122-124

18. Watters E., (2006), DNA Is Not Destiny, in Discover

Online resources

1. http://www.desprecopii.com/info-id-21575-nm-Epigenetica-stiinta-modificarilor-genelor.htm

2. http://www.newscientist.com/article/mg21028126.300-teen-survey-reveals-gene-forhappiness.html

3. http://en.wikipedia.org/wiki/Nature_versus_nurture. 